\title{
Intracerebral Bleeding in Young Infants due to Rare Etiologies-A Report of two Cases
}

\author{
Vishal Vishnu Tewari ${ }^{1 *}$, Kunal Tewari ${ }^{2}$ and Ritu Mehta ${ }^{3}$ \\ ${ }^{1}$ Department of Pediatrics, Army Hospital (Referral and Research), New Delhi, India \\ ${ }^{2}$ Department of Anaesthesia, Classified Specialist Anesthesia, Base Hospital, New Delhi, India \\ ${ }^{3}$ Department of Pathology, All India Institute of Medical Sciences, New Delhi, India
}

*Corresponding author: Vishal Vishnu Tewari, Department of Pediatrics, Army Hospital, New Delhi, India,Tel: +91-8826118889, E-mail: docvvt_13@hotmail.com Rec date: April 29, 2016; Acc date: May 16, 2016; Pub date: May 23, 2016

Copyright: (c) 2016 Tewari VV, et al. This is an open-access article distributed under the terms of the Creative Commons Attribution License, which permits unrestricted use, distribution, and reproduction in any medium, provided the original author and source are credited.

\begin{abstract}
Neonates and young infants are physiologically encumbered by an inadequate hemostatic mechanism. They may also have inherited or acquired bleeding disorders which may have a catastrophic presentation with intracerebral hemorrhage. The need to reach an accurate diagnosis is paramount in order to provide accurate therapy and genetic counseling. We report two infants who presented with unprovoked life threatening massive intracerebral hemorrhage. The first infant was diagnosed and managed for congenital factor $V$ deficiency while the second infant had Glanzmann thrombasthenia.
\end{abstract}

Keywords: Intracerebral bleeding; Congenital factor V deficiency; Glanzmann thrombasthenia

\section{Introduction}

Neonates and young infants have a physiological inadequacy of their coagulation pathway due to lower levels of clotting factors and a deficit in platelet function. In addition inherited or acquired causes of bleeding may present in the neonatal period or early infancy [1]. Hemostasis is an effective but complex process which requires integration of various coagulation factors along with vessel wall integrity. An accurate history and a detailed clinical examination provide clues about the likelihood of a bleeding disorder and whether the abnormality lies in blood vessels, platelets or in the coagulation process [2]. We present two infants who presented with intracerebral hemorrhage due to rare bleeding disorders. The first infant was diagnosed to have congenital factor $\mathrm{V}$ deficiency and second was diagnosed with Glanzmann's thrombasthenia.

\section{Case Report}

\section{Case report 1}

A 19 day old male neonate presented with seizures, encephalopathy and acute onset swelling of right thigh. The seizures semiology was generalized tonic in nature and had been preceded by a short period of irritability, poor feeding and inconsolable crying, followed by altered sensorium and encephalopathy. A few hours later parents noted swelling in the right mid-thigh. Parents reported similar swelling and hematoma formation at the site of Hepatitis-B immunization given at 2 weeks age. The infant was third in birth order and was born to second degree consanguineous couple by normal vaginal route and had weighed $3.8 \mathrm{~kg}$ at birth. The antenatal period had been unremarkable. The first born had been a full-term male infant who had succumbed to an acute illness with jaundice at 3 month age. No details or records were available for scrutiny. The second sibling was a 4 year old female child who was healthy. There was no documented history of bleeding disorder in the family. Antenatal period was uneventful and in the immediate postnatal period the infant had developed unconjugated physiological hyperbilirubinemia. The infant had been well and thriving and weighed $3.9 \mathrm{~kg}$ at admission with an occipito-frontal circumference $(\mathrm{OFC})$ of $36.5 \mathrm{~cm}$. At admission the infant showed paralysis of upward gaze and stridor due to increased intracranial pressure and hindbrain dysfunction (Figure 1).

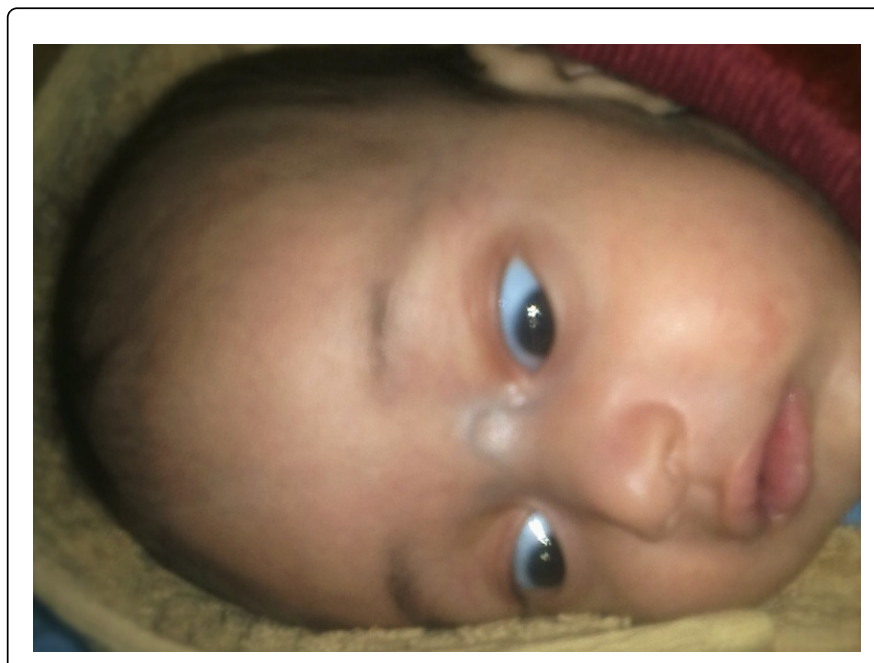

Figure 1: Image of the neonate showing paralysis of upward gaze due to raised intracranial pressure.

The infant was subjected to urgent hematology, biochemistry, sepsis screening and neuroimaging concurrently with measures to control the seizures and maintain cardio-respiratory and hemodynamic status. Baseline biochemistry including the liver function tests was normal. The $\mathrm{Hb}$ was $6.7 \mathrm{~g} / \mathrm{dl}$ and platelet count was $280000 \mathrm{cu} . \mathrm{mm}$. The infection screen was negative. The Contrast enhanced CT scan (CECT) of brain showed intra-parenchymal bleeding with right sided 
intracerebral hematoma and gross midline shift (Figures 2 and 3). Coagulation profile showed prolonged Prothrombin time (PT) and Activated partial thromboplastin time (APTT) with normal platelet count excluding the diagnosis of late-onset vitamin $\mathrm{K}$ deficiency bleeding (VKDB). PT was $79.5 \mathrm{~s}$ (control $19 \mathrm{~s}$ ) and APTT was $127 \mathrm{~s}$ (control $38 \mathrm{~s}$ ). In view of the abnormal PT and APTT, normal platelet count and negative infection screen a coagulation assay was done which included estimation of Factor V, VIII, $\mathrm{X}$ and serum fibrinogen levels. The assay revealed plasma Factor $\mathrm{V}$ levels of $<6 \%$ of normal (normal factor $\mathrm{V}$ level $0.5-1.5 \mathrm{IU} / \mathrm{ml}$ ) with other coagulation factors within normal limits. The infant was diagnosed to have congenital factor $\mathrm{V}$ deficiency and was managed with invasive ventilation, inotropic support, intravenous phenobarbitone, measures to reduce the raised intracranial pressure with 20\% Mannitol and Fresh Frozen plasma (FFP) and Packed Red blood cells (PRBC) transfusions. The infant was taken off ventilator and inotropic support by day-8. Factor $\mathrm{V}$ assay of both parents was indicative of heterozygous factor $\mathrm{V}$ deficiency and carrier state. Genetic analysis was not done as the parents were not willing and the test was not available at our institute. The infant returned for follow-up and was showing slow head growth, delayed milestones and early right hand preference and breakthrough seizures while on oral anti-epileptics.

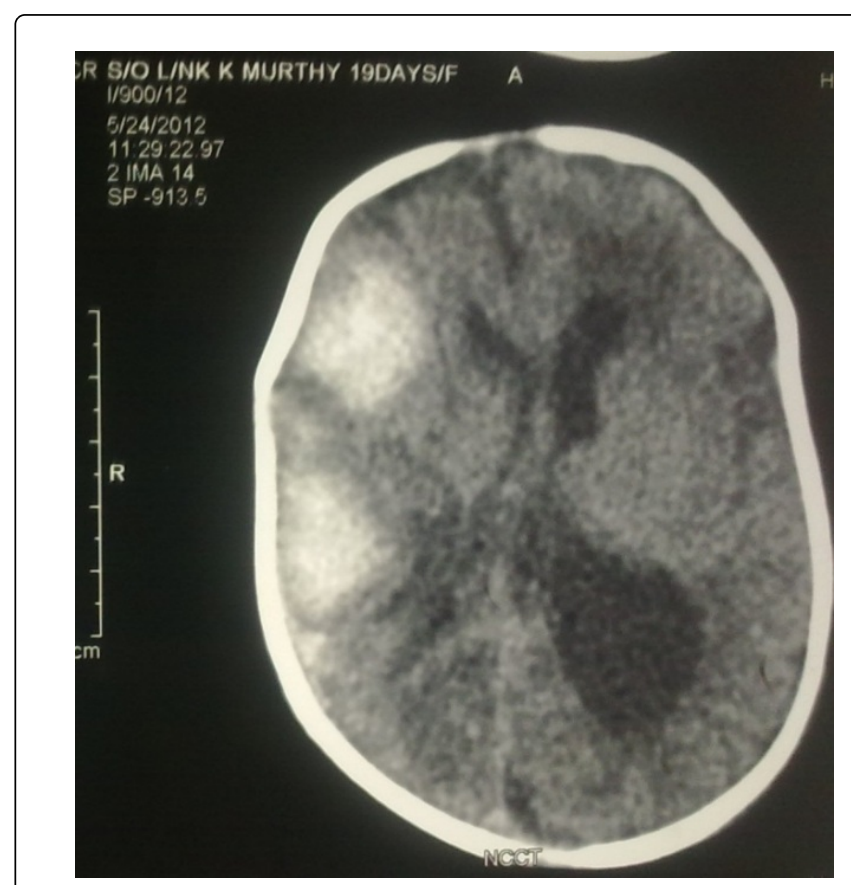

Figure 2: Non-contrast enhanced CT brain with two well delineated hyperdense opacities with surrounding hypodensity on the right side with compression of the ipsilateral ventricle and creating a midline shift.

\section{Case report 2}

A one month old female neonate presented with one day history of unprovoked generalized tonic seizures and status epilepticus. She was born at term to non-consanguineous parents and weighed $2.8 \mathrm{~kg}$. The infant was exclusively breast fed and there was no history of maternal intake of antiepileptics, anti-tubercular drugs or antiplatelet drugs. There was no developmental milestones delay. There was no history of preceding fever, poor feeding, altered sensorium or head trauma. On examination her weight was $4.2 \mathrm{~kg}$, length was $52 \mathrm{~cm}$ and $\mathrm{OFC}$ was $36.2 \mathrm{~cm}$. The anterior fontanelle was $2.5 \times 2.5 \mathrm{~cm}$ and was tense and non-pulsatile. There was no ear discharge, and no petechiae, purpuric spots or ecchymosis. The infant was encephalopathic with tonic posturing and extensor planter response on both sides. The pupils were mid-position and showed sluggish pupillary reaction to light. The Dolls eye reflex was preserved. The infant required intravenous phenobarbitone, fosphenytoin and midazolam infusion to control the seizures. In addition she required assisted ventilation and inotropic support with dopamine infusion for hypotension.

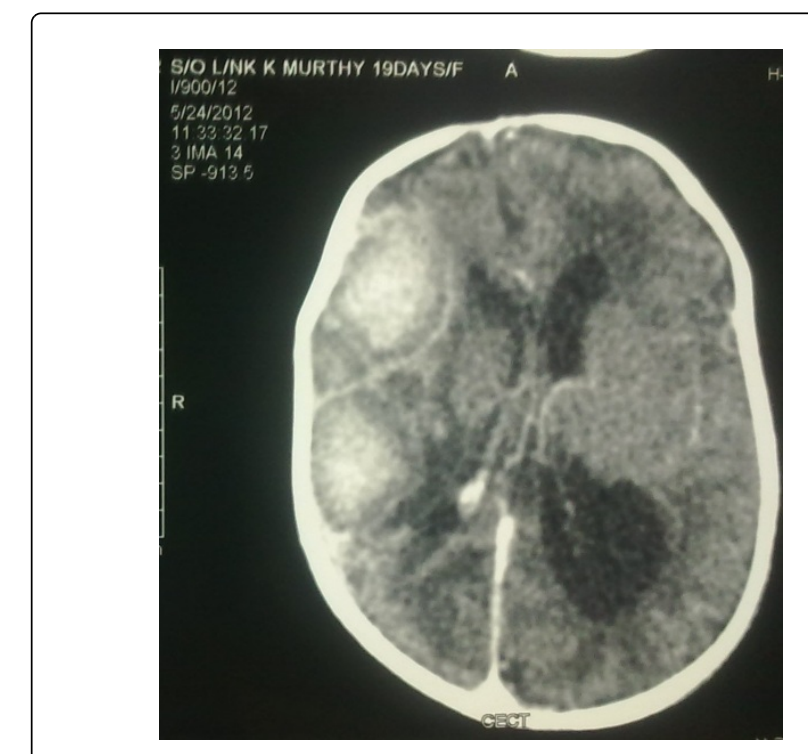

Figure 3: Post-contrast enhancement of the CT brain lesions.

An urgent neuroimaging of the brain with MRI showed the presence of intraparenchymal bleed in the left frontal region and bilateral occipital subdural extension of bleeding with subdural hygroma on the right side (Figures $4 \mathrm{a}$ and $4 \mathrm{~b}$ ). The coronal and sagittal sections delineated the extensive antero-posterior extent and depth of the bleeding (Figures $5 \mathrm{a}$ and $5 \mathrm{~b}$ ). The infant was evaluated for a bleeding diathesis. Her platelet count was 480000 per cu.mm, prothrombin time $13 \mathrm{~s}$ (control $13 \mathrm{~s}$ ), activated partial thromboplastin time was $35 \mathrm{~s}$ (control $30 \mathrm{~s}$ ), thrombin time $21 \mathrm{~s}$ (control $16 \mathrm{~s}$ ). The von Willebrand factor assay was normal. In light of the normal platelet count and coagulogram, testing for platelet functions was resorted to. Bleeding time was $28 \mathrm{~s}$ (control $25 \mathrm{~s}$ ), platelet factor-3 (PF3) availability with adenosine di-phosphate (ADP) at 0 min was $26 \mathrm{~s}$ (control $18 \mathrm{~s}$ ) and at $20 \mathrm{~min}$ was $10 \mathrm{~s}$ (control $8 \mathrm{~s}$ ). Whole blood impedence aggregometry showed that platelet aggregation with ADP, adrenaline (ADR) and arachidonic acid (AA) was reduced while with Ristocetin it was normal. She was diagnosed as a case Glanzmann's thromabasthenia and was given single donor platelet transfusions. She was started on antiepileptic therapy and is on regular follow-up and at age $2 \frac{1}{2}$ years has attained all developmental milestones appropriately reflecting the immense plasticity of the infant brain (Figure 6). 


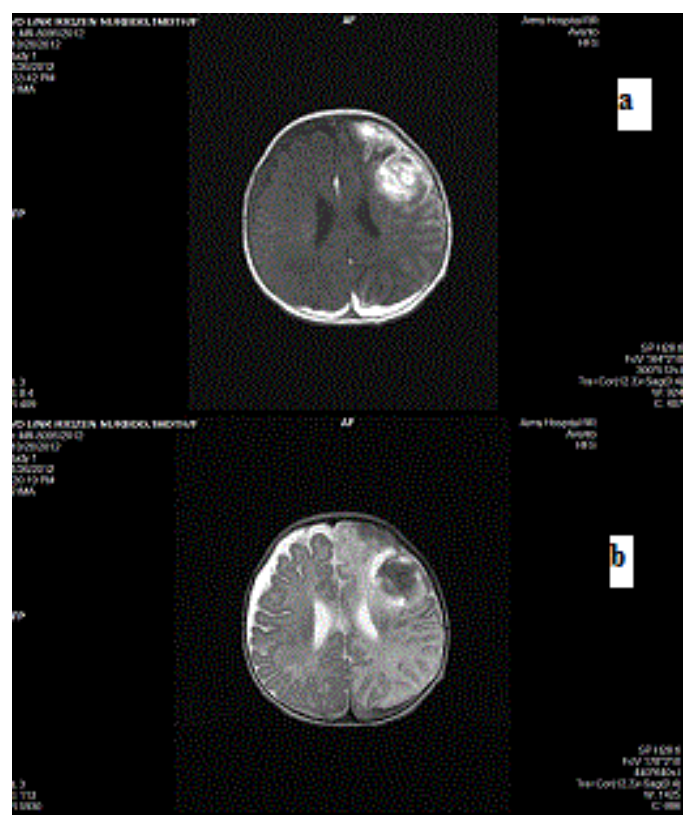

Figure 4a: TI weighted image showing hyperintensity in the left frontal lobe and overlying bilateral occipital lobes due large intracerebral hematoma and bilateral subdural extension of bleed. $b$ : Matched T2 weighted image showing hypointense lesion in the left frontal lobe and hyperintensity overlying the right cerebral hemisphere due to subdural hygroma.

\section{Discussion}

The blood coagulation and fibrinolysis systems are in a unique balance in our body and under tight regulation by inhibitors aiming to contain these systems at the site of injury [3]. Impaired ability to form a clot is the hallmark of a bleeding disorder which may occur spontaneously or following injury. The coagulation process requires interplay of various proteins present in the blood in order to achieve hemostasis which can be defined as the process that requires thrombin formation, fibrin clot formation and fibrin clot dissolution. Bleeding could be due to reduced clotting factors, presence of anticoagulants or due to platelet dysfunction. A detailed clinical history and high index of suspicion is required for diagnosis of congenital bleeding disorders. Symptoms vary from patient to patient and the type of bleeding depends upon the cause off bleeding disorder. Mucosal bleeding, petichiae and easy bruisability may be seen due to platelet disorders or disorders of vessel wall integrity, while intra-articular bleeding, hematomas or intracerebral bleeding reflects possibility of clotting factor deficiency. The investigation of bleeding disorder is a step by step process which includes a complete blood count, peripheral blood smear, PT, APTT, coagulation factors assay, d-dimer, factor inhibitors, fibrinogen, platelet function and aggregation study, Risocetin factor, thrombin time and von Willibrand factor [4]. Our first patient was diagnosed as a congenital factor V deficiency. Factor V is essential for hemostasis and is synthesized in the liver with approximately $80 \%$ circulating in plasma in inactive form and remaining $20 \%$ present within alpha granules of platelets [5].

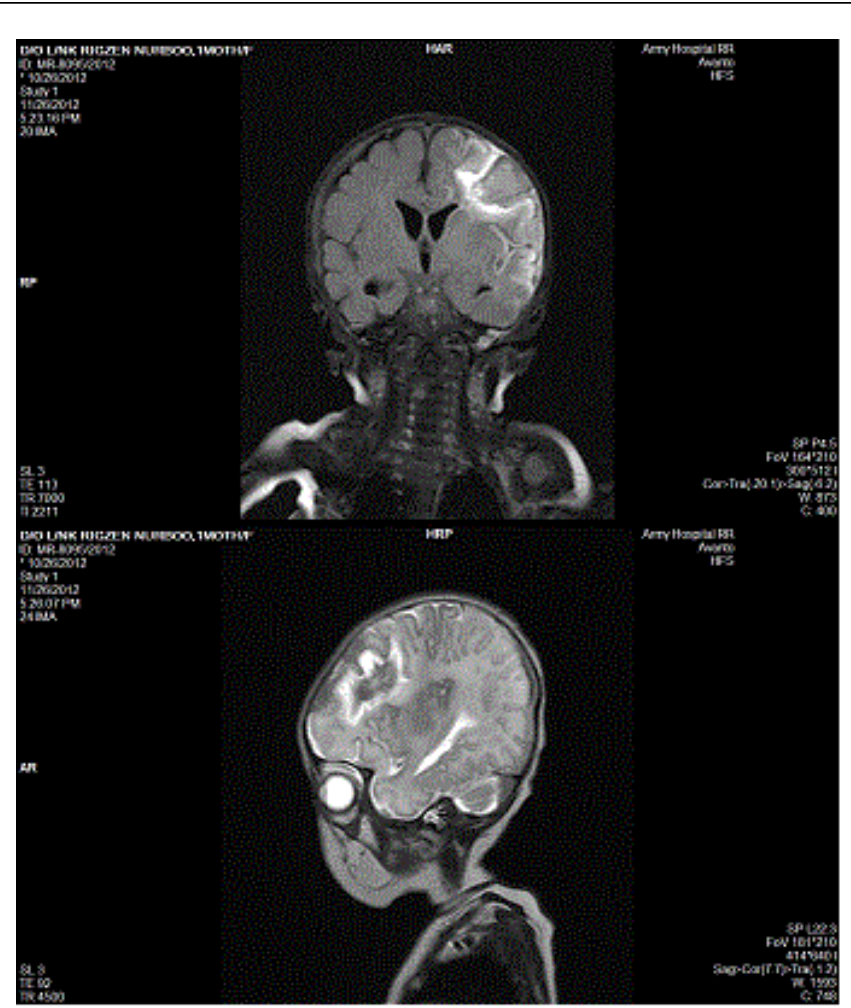

Figure 5a: T1 weighted image in coronal view showing the extent of the bleed and normal ventricular system. b: T2 weighted image in sagittal view showing the antero-posterior extent of the bleed.

Thrombin is required for activation of factor V. Activated factor V is essential for activation of factor $\mathrm{X}$ which in turn is mandatory for conversion of prothrombin to thrombin leading to hemostasis. Congenital factor $\mathrm{V}$ deficiency or parahemophilia is a rare autosomal recessive coagulation disorder also called Owren's disease after Paul Owren who first identified the disorder in $1940[6,7]$ while it was Kingsley who identifed the autosomal recessive pattern of inheritance of deficiency state [8]. Both males and females are affected equally. It is characterized by prolonged PT and APTT with normal bleeding time and platelet count. Congenital factor $\mathrm{V}$ deficiency occurs due to mutations in factor $\mathrm{V}$ gene (F5) itself or in genes that affect the storage and processing of factor $\mathrm{V}$. Mutations of factor $\mathrm{V}$ gene are further subclassified into quantitative (type I) and qualitative (type II). Qualitative (type II) defects are more common. No clear genotype-phenotype correlations have been noted [9]. The age and symptoms of presentation in congenital factor $\mathrm{V}$ deficiency vary from patient to patient. The commonest presenting symptom is bleeding from skin and mucous membranes, followed by bleeding into joints, muscles, genitourinary tract, gastrointestinal tract and central nervous system. Our patient presented with intracerebral bleeding and muscle hematoma. There are limited treatment modalities available. No commercial preparation of factor $\mathrm{V}$ concentrate is currently available. FFP infusion is the mainstay of treatment. Since half-life of factor V is 12-36 h regular infusions are required to maintain hemostasis. Our second infant was diagnosed as a case of Glanzmann thrombasthenia (GT). It is an autosomal recessive disorder which is characterized by quantitative or qualitative abnormalities of the platelet GPIIb and IIIa receptors. The gene for GPIIb and IIIa are located on chromosome 17. 
Page 4 of 4

It binds to fibrinogen, vWF, fibronectin and vitronectin. Genetic defect in GPIIb and IIIa leads to reduced levels of fibrinogen receptors and defective fibrinogen binding after platelet activation [10]. Heterozygous individuals are asymptomatic carriers. Homozygous individuals present shortly after birth with easy tendency to bruise and bleeding. Severe bleeding is common after surgical procedures. Other symptoms are epistaxis, bleeding from the gums, gastrointestinal bleeding, and purpura. The severity of the symptoms varies greatly. Lab investigations show normal platelet count with prolonged bleeding time, deficient clot retraction time and deficient platelet aggregation with ADP, ADR, thrombin and collagen. Ristocetin induced aggregation and other coagulation parameters are normal [11]. Diagnosis based on monoclonal antibodies and flow cytometry to identify GPIIb/GPIIIa receptor deficiency or genetic testing to identify DNA mutations are not routinely available and are not helpful in a life threatening bleed situation like intracerebral bleeding. Treatment is based on the symptoms and mainstay of treatment is single donor platelet transfusions. Recombinant factor VIIa can be used. Treatment is mainly given prior to surgical procedures [12].

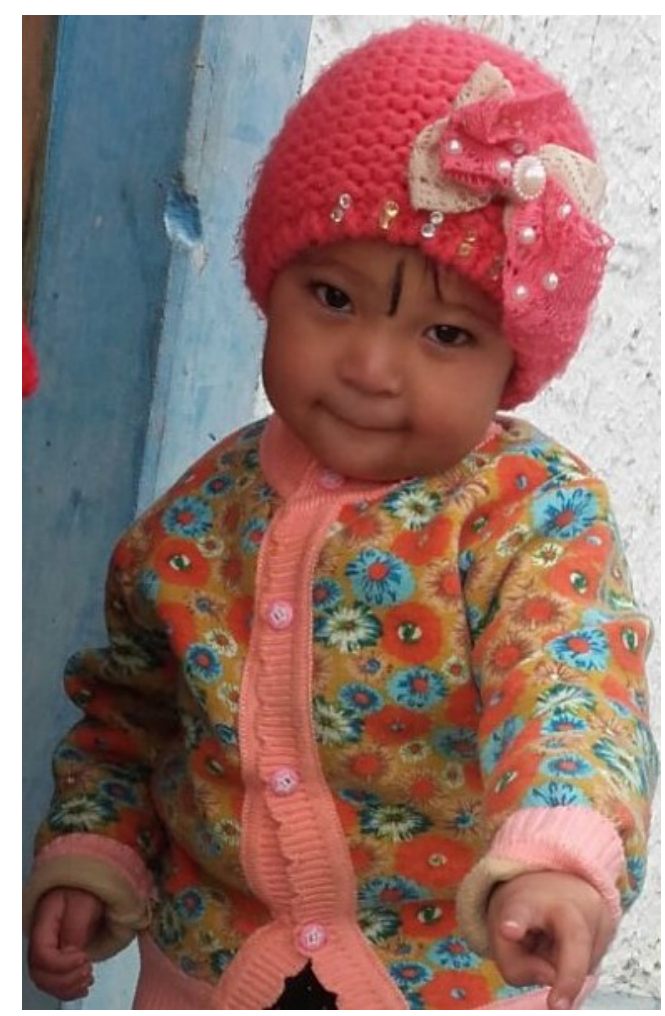

Figure 6: Photograph of the child at age $2 \frac{1}{2} 2$ years showing the child to be interactive and ambulant with age appropriate milestones.

\section{Conclusion}

Bleeding due to rare inherited clotting factor deficiency or platelet function disorders may remain unsuspected and undiagnosed till the patient becomes symptomatic. Central nervous system bleeding is catastrophic in nature and imposes urgency in establishing the diagnosis. A detailed history including family history and examination followed by an algorithmic stepwise investigation allows identification of the disorder to enable directed therapy and genetic counselling.

\section{References}

1. Khair K, Liesner R (2006) Bruising and bleeding in infants and children-a practical approach. Br J Haematol 133: 221-231.

2. Wahlberg T, Blomback M, Hall P, Axelsson G (1980) Application of indicators, predictors and diagnostic indices in coagulation disorders. Methods Inf Med 19: 194-200.

3. Mann KG (1992) Normal hemostasis. (2nd edn), JB Lippincott Co, Philadelphia, London, Toranto.

4. Hayward CPM, Moffat KA, Plumhoff E, Van Cott EM (2012) Approaches to investigating common bleeding disorders: An evaluation of North American coagulation laboratory practices. Am J Hematol 87: S45-S50.

5. Kalafatis M (2005) Coagulation factor V: a plethora of anticoagulant molecules. Curr Opin Hematol 12: 141-148.

6. Asselta R, Tenchini ML, Duga S (2006) Inherited defects of coagulation factor V: the hemorrhagic side. J Thromb Haemost 4: 26-34.

7. Owren PA (1947) Parahaemophilia; haemorrhagic diathesis due to absence of a previously unknown clotting factor. Lancet 1: 446-448.

8. Kingsley CS (1954) Familial factor V deficiency: the pattern of heredity. Q J Med 23: 323-329.

9. Murray JM, Rand MD, Egan JO, Murphy S, Kim HC, et al. (1995) Factor VNew Brunswick: Ala221-to-Val substitution results in reduced cofactor activity. Blood 86: 1820-1827.

10. French DL, Coller BS (1997) Hematologically important mutations: Glanzmann thrombasthenia. Blood Cells Mol Dis 23: 39-51.

11. Mitchell WB, French DL (2003) Glanzmann Thrombasthenia. Lippincott, Williams and Wilkins, Philadelphia, London, Toranto.

12. Di Minno G, Coppola A, Di Minno MN, Poon MC (2009) Glanzmann's thrombasthenia (defective platelet integrin alphaIIb-beta3): proposals for management between evidence and open issues. Thromb Haemost 102: 1157-1164. 